\section{To be, or not to be engaged in sport activities, that is the amletic question for patients with coronary artery disease}

\author{
Silvia Castelletti' and Lia Crotti ${ }^{1,2,3,4}$
}

European Journal of Preventive Cardiology $0(00)$ I-3

(C) The European Society of Cardiology 2019

Article reuse guidelines: sagepub.com/journals-permissions DOI: I0.1 I77/20474873 | 987770 | journals.sagepub.com/home/cpr

(S)AGE
The strong link between physical activity and major non-communicable diseases (NCDs), responsible for $63 \%$ of global deaths, was highlighted during the 66th World Health Assembly endorsed by the World Health Organization (WHO). ${ }^{1}$ Indeed, to improve the prevention of NCDs, WHO launched a global action plan on physical activity, called 'more active people for a healthier world' that should produce a $10 \%$ reduction of physical inactivity by 2025 and a $15 \%$ reduction by 2030 (www.who.int/ncds/prevention/physical-activity/ global-action-plan-2018-2030). Unfortunately, the current trend is going in the opposite direction, with an increase in insufficient physical activity, mainly in high-income countries and also with a significant gender difference, with women being less active than men. $^{2}$ In such a context, the cardiological scientific community has the duty to emphasise the importance of physical activity to reduce all-cause cardiovascular mortality ${ }^{3}$ and also the risk of sudden cardiac death during myocardial infarction, ${ }^{4}$ and to suggest restriction from sports activity only when really needed. This is what was masterfully done by the Sport Cardiology Section of the European Association of Preventive Cardiology (EAPC) which has chosen to publish a document ${ }^{5}$ of recommendations for participation in leisure time or competitive sports in athletes-patients with coronary artery disease (CAD) and in this journal a brief summary of the document. ${ }^{6}$ The summary contains all the main information provided in the original document ${ }^{5}$ with the advantage for the reader of being more schematic and straightforward. They represent an update of the recommendations from the Sports Cardiology section of the EAPC of $2005^{7}$ and are part of a series of sport recommendations in different sets of patients. ${ }^{8-10}$ The choice to update the 2005 document with more disease-specific recommendations has the advantage of grouping more experts for each condition and favours an easier accessibility for the reader. Furthermore, this has allowed a deeper analysis of all conditions potentially causing myocardial ischaemia, including coronary artery abnormalities, spontaneous dissection and myocardial bridging of the coronary arteries, all settings that were not included in the 2005 recommendations.

Regular physical activity is associated with health benefits on cardiovascular function ${ }^{11,12}$ through the modulation of a number of risk factors, including hypertension, body weight, diabetes and cholesterol levels. Its essential role in cardiovascular disease prevention has been emphasised by all leading cardiological societies in their guidelines. ${ }^{13,14}$ Furthermore, even in patients with CAD, leisure time activity has been shown to reduce significantly the risk of cardiac death. ${ }^{15}$ On the other hand, patients with CAD, coronary anomalies or dissection or myocardial bridging may be at increased risk of ischaemia during exercise, when there is an increased oxygen demand and ischaemia could also favour arrhythmic risk in such a context. Therefore, it is quite crucial to identify for each patient what is the level of exercise that could be beneficial and when the risk of adverse events could overcome the advantages.

In such an evaluation, two major pillars should be taken into account, i.e. the kind and intensity of sports activity and the underlying cardiological condition of the athlete. The document by Borjesson ${ }^{6}$ is guiding the physician in such a difficult decision path, not always supported by strong scientific evidence, above all in patients with coronary anomalies, dissection or myocardial bridging, whose diagnosis is increasing substantially with recent technological advances. ${ }^{16}$ Indeed,

IIRCCS Istitito Auxologico Italiano, Center for Cardiac Arrhythmias of Genetic Origin, Milan, Italy

${ }^{2}$ IRCCS Istitito Auxologico Italiano, Laboratory of Cardiovascular Genetics, Milan, Italy

${ }^{3}$ IRCCS Istitito Auxologico Italiano, Department of Cardiovascular, Neural and Metabolic Sciences, Milan, Italy

${ }^{4}$ Department of Medicine and Surgery, University of Milano-Bicocca, Milan, Italy

\section{Corresponding author:}

Silvia Castelletti, Istituto Auxologico Italiano, IRCCS, Centre for Cardiac Arrhythmias of Genetic Origin, via Pier Lombardo 22, 20135 Milan, Italy. Email: s.castelletti@auxologico.it

Twitter: @SilCastelletti 
although we now have a better understanding of the anatomical and pathophysiological consequences of these conditions, the evidence about athletic participation of patients with spontaneous dissection, coronary abnormalities or myocardial bridging is scarce, highlighting the need of further research in these subgroups.

According to this new document, patients with CAD should be restricted from competitive sports only when a substantial risk of adverse events or disease progression is present. This includes asymptomatic patients with CAD and evidence of inducible ischaemia or arrhythmia on functional tests, evidence of myocardial dysfunction, the presence of at least one critical coronary stenosis of a major coronary artery or left main stem, dyspnoea at low exercise intensity or dizziness/ syncope on exertion, or a high degree of myocardial scarring on cardiac magnetic resonance imaging. All other patients, considered at low risk of cardiac events, may be selectively advised to participate in competitive sports; however, as a measure of caution, restrictions may apply for certain sports with the highest cardiovascular demand and for old athletes.

The new section dedicated to non-CAD-related myocardial ischaemia includes patients with congenital coronary artery anomalies (CAA), coronary artery dissection and myocardial bridging. Their inclusion in the document is really important as they are among the most frequent causes of adverse events at younger ages. ${ }^{17}$ Participation in high-intensity sports is discouraged for those patients with CAA originating from the wrong sinus, with acute angled take-off from the aorta and anomalous coursing between the aorta and pulmonary artery. In all other cases, an individualised approach based on comprehensive evaluation focused on assessing the absence of inducible ischaemia is recommended. Athletes-patients with myocardial bridging requiring treatment, even just beta-blockers, should be restricted from participation in competitive sports and should be properly advised regarding leisure-time activities. About patients with coronary dissection, more research has been called for in the document and, for precaution, it is suggested to restrict these patients from intensive sport.

To be picky, a limitation of the current document lies in the few differentiations between competitive and leisure-time activities, essential in countries where the two kinds of sports are differently regulated by countryspecific legislation that affects the practice of the sports physician.

Overall, the document is an invitation to participate in sports, with reasonable precautions, and this is also reflected by the two main terms utilised by the authors throughout the paper: 'coronary artery disease' and 'patients-athletes'. The decision of the authors to replace 'ischaemic heart disease', used in the 2005 document, with 'coronary artery disease' highlights that the presence of coronary disease does not equal an ischaemic disease which instead should be carefully periodically assessed in order to allow the largest number of patients to take part in sports safely. Also the term 'patients-athletes' to define the subjects is a notable choice. This reflects the well-known concept that a healthy lifestyle together with physical activity is a cornerstone not only for primary but also for a patient-tailored secondary prevention. ${ }^{13,15,18}$ Indeed, sport is important in the rehabilitation process; however, individuals who have already experienced ischaemic problems may be more reluctant to engage in physical activity, therefore both athletes and patients who have never been engaged in sports should be reassured through a careful yearly assessment and allowed with reasonable precautions to participate in sports.

In conclusion, sedentary behaviour is a major modifiable risk factor in patients with CAD, not yet adequately treated as clearly outlined by the EUROSPIRE $\mathrm{V}$ results, that showed in more than 8000 patients with CAD that $66 \%$ of them were not physically active. ${ }^{19}$ Clearly, better information/ motivation of the patients is needed, but this must be implemented through an improved education of prescribing physicians. In this setting, the new recommendations for participation in leisure-time or competitive sports in athletes-patients with CAD represent a welcome and timely update, encouraging sports and helping the physician in its prescription.

\section{Declaration of conflicting interests}

The author(s) declared no potential conflicts of interest with respect to the research, authorship, and/or publication of this article.

\section{Funding}

The author(s) received no financial support for the research, authorship, and/or publication of this article.

\section{References}

1. World Health Organization. Global action plan for the prevention and control of noncommunicable diseases: 20132020. 2013. http://apps.who.int/iris/bitstream/10665/ 94384/1/9789241506236_eng.pdf (accessed 9 August 2019).

2. Guthold R, Stevens GA, Riley LM, et al. Worldwide trends in insufficient physical activity from 2001 to 2016: a pooled analysis of 358 population-based surveys with 1.9 million participants. Lancet Global Health 2018; 6: e1077-e1086.

3. Vasankari V, Husu P, Vähä-Ypyä H, et al. Association of objectively measured sedentary behaviour and physical activity with cardiovascular disease risk. Eur J Prev Cardiol 2017; 24: 1311-1318. 
4. De Ferrari GM, Dusi V, Ruffinazzi M, et al. Physical inactivity is a risk factor for primary ventricular fibrillation. J Am Coll Cardiol 2019; 73: 2117-2118.

5. Borjesson M, Dellborg $M$, Niebauer $J$, et al. Recommendations for participation in leisure time or competitive sports in athletes-patients with coronary artery disease: a position statement from the Sports Cardiology Section of the European Association of Preventive Cardiology (EAPC). Eur Heart J 2019; 40: 13-18.

6. Borjesson M, Dellborg M, Niebauer J, et al. Brief recommendations for participation in leisure time or competitive sports in athletes-patients with coronary artery disease: summary of a position statement from the Sports Cardiology Section of the European Association of Preventive Cardiology (EAPC), Eur J Prev Cardiol. Epub ahead of print 12 September 2019. DOI: 10.1177/ 2047487319876186.

7. Pelliccia A, Fagard R, Bjørnstad HH, et al. Recommendations for competitive sports participation in athletes with cardiovascular disease: a consensus document from the Study Group of Sports Cardiology of the Working Group of Cardiac Rehabilitation and Exercise Physiology and the Working Group of Myocardial and Pericardial Diseases of the European Society of Cardiology. Eur Heart J 2005; 26: 1422-1445.

8. Niebauer J, Börjesson $\mathrm{M}$, Carre $\mathrm{F}$, et al. Recommendations for participation in competitive sports of athletes with arterial hypertension: a position statement from the sports cardiology section of the European Association of Preventive Cardiology (EAPC). Eur Heart J 2018; 39: 3664-3671.

9. Pelliccia A, Solberg EE, Papadakis $M$, et al. Recommendations for participation in competitive and leisure time sport in athletes with cardiomyopathies, myocarditis, and pericarditis: position statement of the Sport Cardiology Section of the European Association of Preventive Cardiology (EAPC). Eur Heart J 2019; 40: 19-33.
10. Niebauer J, Börjesson M, Carre F, et al. Brief recommendations for participation in competitive sports of athletes with arterial hypertension: summary of a position statement from the Sports Cardiology Section of the European Association of Preventive Cardiology (EAPC). Eur J Prev Cardiol 2019; 26: 1549-1555.

11. Maessen MF, Eijsvogels TM, Stevens G, et al. Benefits of lifelong exercise training on left ventricular function after myocardial infarction. Eur J Prev Cardiol 2017; 24: 1856-1866.

12. Siasos G, Athanasiou D, Terzis G, et al. Acute effects of different types of aerobic exercise on endothelial function and arterial stiffness. Eur J Prev Cardiol 2016; 23: 1565-1572.

13. Piepoli MF and Villani GQ. Lifestyle modification in secondary prevention. Eur J Prev Cardiol 2017; 24(3_Suppl): 101-107.

14. Riegel B, Moser DK, Buck HG, et al. Self-care for the prevention and management of cardiovascular disease and stroke: a scientific statement for healthcare professionals from the American Heart Association. $J$ Am Heart Assoc 2017; 6: e006997.

15. Lahtinen M, Toukola $\mathrm{T}$, Junttila MJ, et al. Effect of changes in physical activity on risk for cardiac death in patients with coronary artery disease. Am J Cardiol 2018; 121: 143-148.

16. Lee MS and Chen C-H. Myocardial bridging: an up-todate review. J Invasive Cardiol 2015; 27: 521-528.

17. Corrado D, Basso C, Rizzoli G, et al. Does sports activity enhance the risk of sudden death in adolescents and young adults? J Am Coll Cardiol 2003; 42: 1959-1963.

18. Cortés-Beringola A, Fitzsimons D, Pelliccia A, et al. Planning secondary prevention: room for improvement. Eur J Prev Cardiol 2017; 24(3_Suppl): 22-28.

19. Kotseva K, De Backer G, De Bacquer D, et al. Lifestyle and impact on cardiovascular risk factor control in coronary patients across 27 countries: results from the European Society of Cardiology ESC-EORP EUROASPIRE V registry. Eur J Prev Cardiol 2019; 26: 824-835. 